Reprints in Theory and Applications of Categories, No. 14, 2005, pp. 1-18.

\title{
ENRICHED CATEGORIES AND COHOMOLOGY
}

\author{
To Margery, who typed the original preprint in Milan
}

\author{
ROSS STREET
}

\section{Author Commentary}

From the outset, the theories of ordinary categories and of additive categories were developed in parallel. Indeed additive category theory was dominant in the early days. By additivity for a category I mean that each set of morphisms between two objects (each "hom") is equipped with the structure of abelian group and composition on either side, with any morphism, distributes over addition: that is to say, the category is enriched in the monoidal category of abelian groups. "Enrichment" in this context is happening to the homs of the category. This enrichment in abelian groups is rather atypical since, for a category with finite products or finite coproducts, it is a property of the category rather than a structure.

Linton, in [14], began developing the theory of categories enriched in monoidal categories of sets with structure. Independently of each other, Eilenberg and Kelly recognized the need for studying categories enriched in the monoidal category of chain complexes of abelian groups: differential graded categories (or DG-categories). This led to the collaboration [6] which began the theory of categories enriched in a general monoidal category, called the base.

Soon after, in [1], Bénabou defined bicategories and morphisms between them. He observed that a bicategory with one object is the same as a monoidal category. He noted that a morphism of bicategories from the category $\mathbf{1}$ to Cat is what had been called (after [7]) a category together with a triple thereon; Bénabou called this a monad. For any set $X$ he defined the term polyad in a bicategory $\mathcal{W}$ to mean a morphism from the chaotic category on $X$ to $\mathcal{W}$. This is important here since such a polyad is precisely a category $\mathcal{A}$ enriched in the bicategory $\mathcal{W}$ where the set of objects of $\mathcal{A}$ is $X$.

Categories enriched in $\mathcal{V}$ are closely related to categories on which a monoidal category $\mathcal{V}$ acts (lately called "actegories" [15]) and the latter subject was pursued by Bénabou (in lectures I attended at Tulane University in 1969-70).

Received by the editors 2005-11-16.

Transmitted by G. Max Kelly, R.F.C. Walters and R.J. Wood. Reprint published on 2005-12-31.

2000 Mathematics Subject Classification: 18D20, 18D30.

Originally published as: Enriched categories and cohomology, Quaestiones Mathematicae, 6 (1983), 265-283, used by permission. Paper read at the Symposium on Categorical Algebra and Topology, University of Cape Town, 29 June - 3 July 1981. 
The theory of categories enriched in a complete, cocomplete, symmetric closed monoidal category was developed over the next decade in the pioneering works: [8], [5], [12], [13], [2]. In particular, the last two works discussed a concept that goes under various names: profunctor, bimodule, distributor, or (two-sided) module. In terms of these, Lawvere defined cauchy completeness of an enriched category generalizing the classical notion for metric spaces.

Then came a major advance which meant that categories enriched in a bicategory needed serious consideration. Walters [17] showed that sheaves were such categories. The bicategories appropriate to capture sheaves on a Grothendieck site had hom-categories that were ordered sets.

This is the point at which the present paper was written. The intention was to provide the foundations of the theory of categories enriched in a bicategory whose hom-categories were complete and cocomplete, and which admitted right liftings and right extensions (that is, which was "biclosed" in the sense of Lawvere [12]). The new aspects of the present work included:

- no assumption on symmetry of the base;

- no assumption that the hom-categories of the base bicategory be ordered; and,

- the application to the theory of torsors and cohomology.

There have been many developments of enriched category since the present paper. In Milan in 1981, I asked when two bicategories can have essentially the same categories enriched over them: an answer appears in [16]. The third paper [3] from this period constructs colimits of enriched categories (extending [18]) and proves a precise result showing that categories fibred over a base are enriched over a bicategory.

Meanwhile, Kelly was developing the theory of categories enriched over a symmetric monoidal category; for example, the paper [9] extends the theory of locally presentable categories. Of course, then came Kelly's book [10] which still remains the primary reference when the base is a symmetric monoidal category. The book constructs colimit completions with respect to prescribed weights. Modules only appear implicitly.

A substantial amount of beautiful enriched category theory, with associated application, is surely still to be uncovered. Enrichment in bicategories on two sides (see [11]) provides one new direction, allowing the possibility of composition of enrichments. For applications, a growing source is homotopy theory, where categories enriched in topological spaces and in simplicial sets have been studied for decades; for example, see [4] and the references therein.

However, the subject of this paper may eventually be better understood as occupying a small yet significant corner of higher-dimensional category theory.

I am indebted to Elango Panchadcharam for taking charge of TeXing this paper and to Craig Pastro and Steve Lack for contributions to that process. 


\section{References}

[1] J. Bénabou, Introduction to bicategories, Lecture Notes in Mathematics (SpringerVerlag, Berlin) 47 (1967), 1-77.

[2] J. Bénabou. Les distributeurs. Département de mathématique, Université Catholique de Louvain, Louvain-la-neuve. 33, typed lecture notes (1973).

[3] R. Betti, A. Carboni, R. Street and R. F. Walters, Variation through enrichment, Journal of Pure and Applied Algebra 29 (1983), 109-127. MR 85e:18005

[4] J. Cordier and T. Porter, Homotopy coherent category theory, Transactions of the American Mathematical Society 349 (1997), 1-54. MR 97d:55032

[5] B. J. Day and G. M. Kelly, Enriched functor categories, Lecture Notes in Mathematics 106 (1969), 178-191. MR 41:293

[6] S. Eilenberg and G. M. Kelly, Closed categories, Proc. Conf. Categorical Algebra (La Jolla, Calif., 1965), Springer-Verlag, New York, 1966, 421-562. MR 37:1432

[7] S. Eilenberg and G. Moore, Adjoint functors and triples, Illinois Journal of Mathematics 9 (1965), 381-398.

[8] G. M. Kelly, Adjunction for enriched categories, Lecture Notes in Mathematics 106 (1969), 166-177. MR 41:292

[9] G. M. Kelly, Structures defined by finite limits in the enriched context. I. , Cahiers Topologie Géometrie Différentielle 23 (1982), 3-42. MR 83h:18007

[10] G. M. Kelly, Basic concepts of enriched category theory, London Mathematical Society Lecture Note Series, 64. Cambridge University Press, Cambridge, 1982, and Reprints in Theory and Applications of Categories, no. 10 (2005), 1-136. MR 84e: 18001

[11] M. Kelly, A. Labella, V. Smith and R. Street, Categories enriched on two sides, Journal of Pure and Applied Algebra 168 (2002), 53-98.

[12] F. W. Lawvere (1971). Closed categories and biclosed bicategories. Mathematics Institute Aarhus Universitet, Aarhus. handwritten lecture notes

[13] F. W. Lawvere, Metric spaces, generalized logic and closed categories, Rendiconti del Seminario Matematico e Fisico di Milano,135-166. 43 (1973), 135-166, and Reprints in Theory and Applications of Categories, no. 1 (2002), 1-37. MR 50:4701

[14] F. E. Linton, Autonomous categories and duality of functors, Journal of Algebra 2 (1965), 315-349. MR 31:4821 
[15] P. McCrudden, Categories of representations of coalgebroids, Advances in Mathematics 154 (2000), 299-332.

[16] R. Street, Cauchy characterization of enriched categories, Rendiconti del Seminario Matematico e Fisico di Milano 51 (1981), 217-233, and Reprints in Theory and Applications of Categories, no. 4 (2004), 1-16. MR 85e:18006

[17] R. F. Walters, Sheaves on sites as Cauchy-complete categories, Journal of Pure and Applied Algebra 24 (1982), 95-102.

[18] H. Wolff, $V$-cat and $V$-graph., Journal of Pure and Applied Algebra 4 (1974), 123135. MR 49:10755 


\section{Introduction}

Giraud [Gir] uses the notion of stack (= champ in French) in the construction of nonabelian cohomology. A stack is a certain kind of fibration over a topos $\mathscr{E}$ : a descent condition is to be satisfied. The viewpoint that a fibration over $\mathscr{E}$ is an $\mathscr{E}$-enriched category of models in $\mathscr{E}$ of some theory has been further developed by Lawvere [Law], Bénabou [Bé2]; a slightly different viewpoint is taken by Penon [Pen], Paré-Schumacher [PS]; and a more general setting provided in Street-Walters [SW], Street [St1] (see Street [St2]). Basically, the category of $U$-indexed families of models of the theory in $\mathscr{E}$ becomes the fibre of the fibration over the object $U$ of $\mathscr{E}$. This fibre is a category with homs enriched in the cartesian closed category $\mathscr{E} / U$ in the sense of Eilenberg-Kelly [EK]. It is important to consider the fibration itself and not merely the fibre over the terminal object so that it appears that the theory of hom-enriched categories is not applicable.

In fact, a variant of the Eilenberg-Kelly theory does apply. It was pointed out by Bénabou [Bé1] that a monoidal category $\mathscr{V}$ is a bicategory with one object and that it is possible to define categories enriched in a bicategory (he called them "polyads"). Little work seems to have been done on this concept. Betti [Bt1] discovered examples in automata theory. Walters [Wal] discovered that sheaves on a site are precisely Cauchycomplete, symmetric categories enriched in a relations-like bicategory determined by the site. This has activated work on the subject by Betti [Bt2], Betti-Carboni [BC]; however, their interest has been predominantly in bicategories whose hom-categories are posets.

To be relevant to cohomology, enriched category theory must be developed over a base bicategory which does not necessarily have posetal homs. Aspects of this theory are presented here with an indication of its relevance to cohomology.

\section{Categories enriched over a bicategory}

Let $\mathscr{W}$ denote a bicategory in the sense of Bénabou [Bé1]. Recall that a diagram

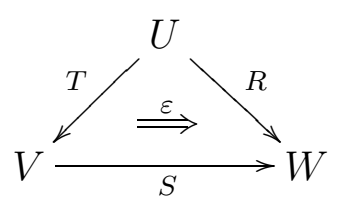

is said to exhibit $T$ as a right lifting of $R$ through $S$ when each 2-cell $\theta: S T^{\prime} \longrightarrow R$ factors as $\varepsilon \circ S \tilde{\theta}$ for a unique 2-cell $\tilde{\theta}: T^{\prime} \longrightarrow T$. We write $S \pitchfork R$ for a particular choice of right lifting of $\mathrm{R}$ through $\mathrm{S}$.

We say $T: U \longrightarrow V$ is right adjoint to $S: V \longrightarrow T$ with counit $\varepsilon: S T \longrightarrow 1_{U}$ when $\tilde{\varepsilon}: T \cong S \pitchfork 1_{U}$ and $\widetilde{\varepsilon S}: T S \cong S \pitchfork S$. An arrow $S$ in $\mathscr{W}$ which has a right adjoint $T$ is called a map in $\mathscr{W}$. Maps will be denoted by lower case letters such as $f: V \longrightarrow U$; its right adjoint will be denoted $f^{*}: U \longrightarrow V$; and the counit will be denoted by $\varepsilon_{f}$.

A $\mathscr{W}$-category $\mathscr{A}$ consists of the following data:

- for each object $U$ of $\mathscr{W}$, a set $\mathscr{A}_{U}$ of objects over $U$; 
- for objects $A, B$ over $U, V$, respectively, an arrow $\mathscr{A}(B, A): U \longrightarrow V$ in $\mathscr{W}$;

- for objects $A, B, C$ over $U, V, W$, respectively, 2-cells

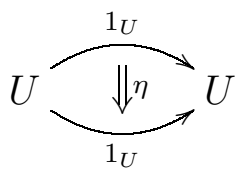

and

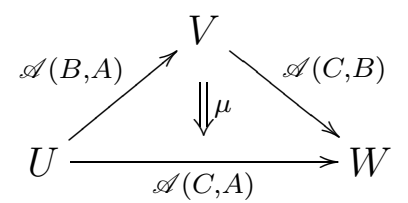

in $\mathscr{W}$;

satisfying the obvious three axioms of left and right identities and associativity.

If $A$ is an object of $\mathscr{A}$ over $U$ it is convenient to write $e A=U$. In fact, the assignment $A \longmapsto e A$ extends to a morphism of bicategories (= lax functor) from the chaotic category on the set of objects of $\mathscr{A}$ to $\mathscr{W}$ (Bénabou's polyad).

A $\mathscr{W}$-category $\mathscr{A}$ with precisely one object $A$ amounts to a monad in $\mathscr{W}$ made up of the object $e A$ of $\mathscr{W}$ and endo-arrow $\mathscr{A}(A, A): e A \longrightarrow e A$. (Actually a $\mathscr{W}$-category is precisely a monad in an appropriate bicategory, but this point of view will not be needed here.) Each object $U$ of $\mathscr{W}$ will be identified with the $\mathscr{W}$-category which amounts to the identity monad on $U$.

2.1. ExAmple. Suppose $\mathscr{E}$ is a finitely complete category for which each of the comma categories $\mathscr{E} / U$ is cartesian closed (we call such a category internally complete). Let $\mathscr{W}=$ Spn $\mathscr{E}$ be the bicategory of spans in $\mathscr{E}$ : the objects are those of $\mathscr{E}$, the arrows $S: U \longrightarrow V$ are spans from $U$ to $V$ so that $\mathscr{W}(U, V)$ is essentially $\mathscr{E} / U \times V$, and composition is given by pullback. Any two arrows in $\mathscr{W}$ with a common target admit a right lifting of one through the other.

Let $\mathscr{F}$ be a fibration over $\mathscr{E}$ localement petite in the sense of Bénabou [Bé2]. We can define a $\mathscr{W}$-category $\mathscr{X}$ as follows. The objects of $\mathscr{X}$ over $U$ are objects of the fibre of $\mathscr{F}$ over $U$. If $X, Y$ are objects over $U, V$ then $\mathscr{X}(Y, X): U \longrightarrow V$ is the (unique up to isomorphism) span with the property that arrows of spans

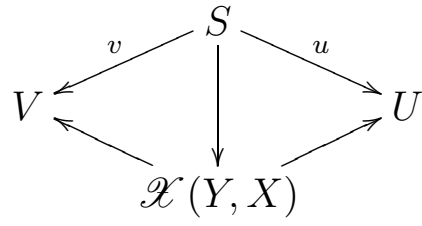

are in natural bijection with arrows $u^{X} \longrightarrow v^{Y}$ in the fibre of $\mathscr{F}$ over $S$.

A $\mathscr{W}$-functor $F: \mathscr{A} \longrightarrow \mathscr{C}$ consists of the following data:

- a function which assigns to each object $A$ of $\mathscr{A}$ over $U$, an object $F A$ of $\mathscr{C}$ over $U$;

- for objects $A, B$ of $\mathscr{A}$ over $U, V$, a 2-cell

$$
F_{A B}: \mathscr{A}(B, A) \longrightarrow \mathscr{C}(F B, F A)
$$

in $\mathscr{W}$; 
satisfying the obvious conditions on preservation of $\eta, \mu$.

For any object $U$ of $\mathscr{W}$, a $\mathscr{W}$-functor $U \longrightarrow \mathscr{X}$ precisely amounts to an object of $\mathscr{X}$ over $U$, and we freely use this identification.

Notice that, for each object $U$ of $\mathscr{W}$, composition in $\mathscr{W}$ determines a tensor product on $\mathscr{W}(U, U)$ yielding a monoidal category. If $\mathscr{A}$ is a $\mathscr{W}$-category, the objects of $\mathscr{A}$ over $U$ determine a $\mathscr{W}(U, U)$-category $\mathscr{A}_{U}$. There is an underlying category for $\mathscr{A}_{U}$ : an arrow $f: A \longrightarrow B$ in $\mathscr{A}_{U}$ is a 2 -cell $1_{U} \longrightarrow \mathscr{A}(A, B)$ in $\mathscr{W}$. For any object $C$ of $\mathscr{A}$, we can define 2-cells:

- $\mathscr{A}(C, f): \mathscr{A}(C, A) \longrightarrow \mathscr{A}(C, B)$

- $\mathscr{A}(f, C): \mathscr{A}(B, C) \longrightarrow \mathscr{A}(A, C)$

to be the composites:

- $\mathscr{A}(C, A) \stackrel{\mathscr{A}(C, A) f}{\longrightarrow} \mathscr{A}(C, A) \mathscr{A}(A, B) \stackrel{\mu}{\longrightarrow} \mathscr{A}(C, B)$

- $\mathscr{A}(B, C) \stackrel{f \mathscr{A}(B, C)}{\longrightarrow} \mathscr{A}(A, B) \mathscr{A}(B, C) \stackrel{\mu}{\longrightarrow} \mathscr{A}(A, C)$.

Suppose $F, G: \mathscr{A} \longrightarrow \mathscr{C}$ are $\mathscr{W}$-functors. A $\mathscr{W}$-natural transformation $\theta: F \longrightarrow G$ assigns to each object $A$ of $\mathscr{A}$ over $U$, an arrow $\theta_{A}: F A \longrightarrow G A$ in the underlying category of $\mathscr{A}_{U}$, such that the following diagram commutes.

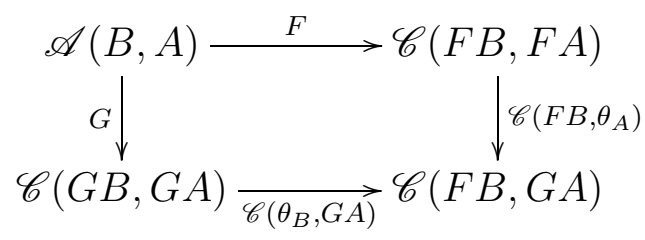

There is a 2 -category $\mathscr{W}$-Cat of $\mathscr{W}$-categories, $\mathscr{W}$-functors, and $\mathscr{W}$-natural transformations. Compositions are defined precisely as for the case where $\mathscr{W}$ is a monoidal category.

\section{Modules}

Henceforth assume that $\mathscr{W}$ and $\mathscr{W}^{\text {op }}$ both admit all right liftings. Then composition in $\mathscr{W}$ preserves all colimits which exist in the hom-categories.

Suppose $\mathscr{A}, \mathscr{C}$ are $\mathscr{W}$-categories. A $\mathscr{W}$-module $\Phi: \mathscr{A} \longrightarrow \mathscr{C}$ assigns:

- to each pair $A, C$ of objects of $\mathscr{A}, \mathscr{C}$ over $U, V$, respectively, an arrow $\Phi(C, A)$ : $U \longrightarrow V$ in $\mathscr{W}$;

- to objects $A, A^{\prime}$ of $\mathscr{A}$ and $C$ of $\mathscr{C}$, a 2-cell $\rho: \Phi\left(C, A^{\prime}\right) \mathscr{A}\left(A^{\prime}, A\right) \longrightarrow \Phi(C, A)$;

- to objects $A$ of $\mathscr{A}$ and $C, C^{\prime}$ of $\mathscr{C}$, a 2-cell $\lambda: \mathscr{C}\left(C^{\prime}, C\right) \Phi(C, A) \longrightarrow \Phi\left(C^{\prime}, A\right)$; 
satisfying the five axioms expressing compatibility of $\rho$ with $\eta, \mu$ in $\mathscr{A}$, of $\lambda$ with $\eta, \mu$ in $\mathscr{C}$, and of $\rho, \lambda$ with each other.

Each pair of $\mathscr{W}$-functors $J: \mathscr{A} \longrightarrow \mathscr{X}, K: \mathscr{C} \longrightarrow \mathscr{X}$ determine a $\mathscr{W}$-module from $\mathscr{A}$ to $\mathscr{C}$, which we denote by $\mathscr{X}(K, J)$, as follows:

- $\mathscr{X}(K, J)(C, A)=\mathscr{X}(K C, J A)$;

- $\rho: \mathscr{X}\left(K C, J A^{\prime}\right) \mathscr{A}\left(A^{\prime}, A\right) \stackrel{\mathscr{X}\left(K C, J A^{\prime}\right) J_{A A^{\prime}}}{\longrightarrow} \mathscr{X}\left(K C, J A^{\prime}\right) \mathscr{X}\left(J A^{\prime}, J A\right) \stackrel{\mu}{\longrightarrow} \mathscr{X}(K C, J A)$;

- $\lambda: \mathscr{C}\left(C^{\prime}, C\right) \mathscr{X}(K C, J A) \stackrel{K_{C C^{\prime}} \mathscr{X}(K C, J A)}{\longrightarrow} \mathscr{X}(K C, K C) \mathscr{X}(K C, J A) \stackrel{\mu}{\rightarrow} \mathscr{X}\left(K C^{\prime}, J A\right)$;

In particular, a $\mathscr{W}$-functor $F: \mathscr{A} \longrightarrow \mathscr{C}$ determines $\mathscr{W}$-modules $F_{*}=\mathscr{C}\left(1_{\mathscr{C}}, F\right): \mathscr{A} \longrightarrow \mathscr{C}$ and $F^{*}=\mathscr{C}\left(F, 1_{\mathscr{C}}\right): \mathscr{C} \longrightarrow \mathscr{A}$.

For $\mathscr{W}$-modules $\Phi, \Psi: \mathscr{A} \longrightarrow \mathscr{C}$, a 2-cell $\theta: \Phi \longrightarrow \Psi$ is a family of 2-cells $\theta_{A C}$ : $\Phi(C, A) \longrightarrow \Psi(C, A)$ in $\mathscr{W}$ compatible with the left and right actions $\lambda, \rho$.

For $\mathscr{W}$-functors $F, G: \mathscr{A} \longrightarrow \mathscr{C}$, there are natural bijections between 2-cells $F_{*} \longrightarrow G_{*}$, 2-cells $G^{*} \longrightarrow F^{*}$, and $\mathscr{W}$-natural transformations $F \longrightarrow G$.

Define the composite $\Psi \Phi: \mathscr{A} \longrightarrow \mathscr{X}$ of $\mathscr{W}$-modules $\Phi: \mathscr{A} \longrightarrow \mathscr{C}, \Psi: \mathscr{C} \longrightarrow \mathscr{X}$ as follows:

$(\Psi \Phi)(X, A)$ is the colimit in $\mathscr{W}(e A, e X)$ of the diagram

$$
\Psi(X, C) \Phi(C, A) \stackrel{\rho \Phi(C, A)}{\longleftarrow} \Psi\left(X, C^{\prime}\right) \mathscr{C}\left(C^{\prime}, C\right) \Phi(C, A) \stackrel{\Psi\left(X, C^{\prime}\right) \lambda}{\longrightarrow} \Psi\left(X, C^{\prime}\right) \Phi\left(C^{\prime}, A\right)
$$

in which $C, C^{\prime}$ vary over all the objects of $\mathscr{C}$;

- the $\rho$ for $\Psi \Phi$ is induced by the $\rho$ for $\Phi$;

- the $\lambda$ for $\Psi \Phi$ is induced by the $\lambda$ for $\Psi$.

Of course, the colimit involved in this definition may not exist. There are special circumstances where it does without any cocompleteness assumptions on the hom-categories of $\mathscr{W}$ : for example, we always have $\Phi G_{*}$ and $H^{*} \Psi$ for $\mathscr{W}$-functors $G, H$. By restricting to small enough $\mathscr{W}$-categories, we obtain a bicategory $\mathscr{W}$-Mod of $\mathscr{W}$-categories, $\mathscr{W}$-modules, and 2-cells between them.

It is thus possible to speak of adjoint $\mathscr{W}$-modules. In fact, even without the existence of the colimits needed for composition, it is possible to give meaning to $\Phi \dashv \Psi$ but this leads us into the world of the "pro-bicategories" of Day [Day] and this is too far afield. It is worth remarking however that, for each $\mathscr{W}$-functor $F: \mathscr{A} \longrightarrow \mathscr{C}$, we do have $F_{*} \dashv F^{*}$ where the unit consists of the family of 2-cells $F_{A B}$.

For a $\mathscr{W}$-category $\mathscr{A}$, we shall describe a $\mathscr{W}$-category $\mathcal{P} \mathscr{A}$ which classifies $\mathscr{W}$-modules in the following sense:

$$
\mathscr{W}-\operatorname{Cat}(\mathscr{C}, \mathcal{P} \mathscr{A}) \cong \mathscr{W}-\operatorname{Mod}(\mathscr{C}, \mathscr{A})
$$


Taking $\mathscr{C}$ to be an object $U$ of $\mathscr{W}$, we see that the objects of $\mathcal{P} \mathscr{A}$ over $U$ must be $\mathscr{W}$ modules from $U$ to $\mathscr{A}$. The data for such a $\mathscr{W}$-module $\Phi$ can be expressed in a diagram:

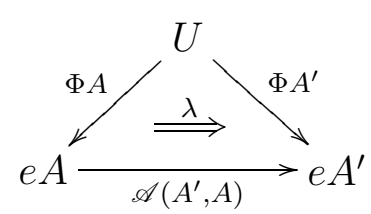

If $\Phi, \Psi$ are objects of $\mathcal{P} \mathscr{A}$ over $U, V$, respectively, then $(\mathcal{P} \mathscr{A})(\Psi, \Phi)$ is defined to be the limit in $\mathscr{W}(U, V)$ of the following diagram as $A, A^{\prime}$ run over all objects of $\mathscr{A}$.

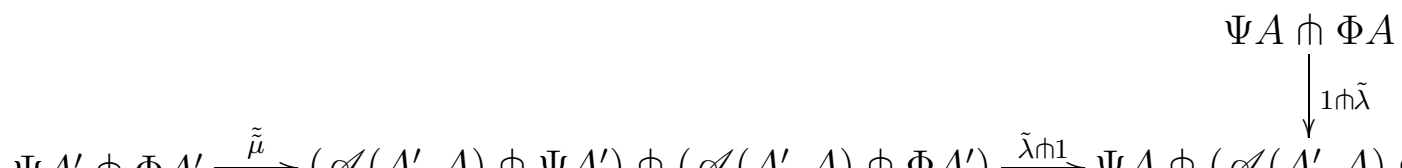

$\Psi A^{\prime} \pitchfork \Phi A^{\prime} \stackrel{\tilde{\tilde{\mu}}}{\longrightarrow}\left(\mathscr{A}\left(A^{\prime}, A\right) \pitchfork \Psi A^{\prime}\right) \pitchfork\left(\mathscr{A}\left(A^{\prime}, A\right) \pitchfork \Phi A^{\prime}\right) \stackrel{\tilde{\lambda} \pitchfork 1}{\longrightarrow} \Psi A \pitchfork\left(\mathscr{A}\left(A^{\prime}, A\right) \pitchfork \Phi A^{\prime}\right)$

Again it may happen that this limit does not exist. Sometimes it does exist free of charge: for example,

$$
(\mathcal{P} \mathscr{A})\left(A^{*}, \Phi\right) \cong \Phi A,
$$

by which we mean that the right-hand side admits a limiting cone for the defining diagram of the left-hand side.

Suppose $\mathscr{A}$ is a $\mathscr{W}$-category with one object $A$, so that we have a $\operatorname{monad} M=\mathscr{A}(A, A)$ on $W=e A$ in $\mathscr{W}$. Then $(\mathcal{P} \mathscr{A})_{U}$ is precisely the $\mathscr{W}(U, U)$-category of $M$-algebras:

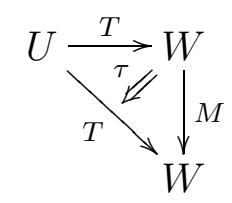

for the monad $\mathscr{W}(U, M)$ on the $\mathscr{W}(U, U)$-category $\mathscr{W}(U, W)$. In this case the limit required in $\mathscr{W}(U, U)$ is a mere equalizer.

The identity $\mathscr{W}$-module of $\mathscr{A}$ corresponds to the Yoneda embedding $Y_{\mathscr{A}}: \mathscr{A} \rightarrow \mathcal{P} \mathscr{A}$ given by:

$$
Y_{\mathscr{A}} A=A_{*^{\prime}} \quad Y_{\mathscr{A} A A^{\prime}}: \mathscr{A}\left(A^{\prime}, A\right) \cong(\mathcal{P} \mathscr{A})\left(A_{*}^{\prime}, A_{*}\right) .
$$

There is also a $\mathscr{W}$-category $\mathcal{P}^{\dagger} \mathscr{A}$ which classifies $\mathscr{W}$-modules in the following sense:

$$
\mathscr{W}-\operatorname{Cat}\left(\mathscr{C}, \mathcal{P}^{\dagger} \mathscr{A}\right) \simeq \mathscr{W}-\operatorname{Mod}(\mathscr{A}, \mathscr{C})^{\mathrm{op}},
$$

provided that the necessary limits exist in the hom-categories of $\mathscr{W}$; the construction is similar to that of $\mathcal{P} \mathscr{A}$. Notice:

$$
\mathscr{W}-\operatorname{Cat}^{\mathrm{coop}}\left(\mathcal{P}^{\dagger} \mathscr{A}, \mathscr{C}\right) \simeq \mathscr{W}-\operatorname{Cat}(\mathscr{A}, \mathcal{P} \mathscr{C})
$$




\section{Cocompleteness}

Henceforth the terms functor, module, natural transformation, will be used in place of the terms with the prefix " $\mathscr{W}-$ " when they apply to $\mathscr{W}$-categories.

A module $\Phi: \mathscr{A} \longrightarrow \mathscr{C}$ is said to converge to a functor $F: \mathscr{A} \longrightarrow \mathscr{C}$ when $\Phi \cong F_{*}$. Since the homomorphism $\mathscr{W}$-Cat $\longrightarrow \mathscr{W}$-Mod which takes $F$ to $F_{*}$ is fully faithful on hom-categories, such an $F$ is unique up to isomorphism if it exists. A module $\Phi$ is called cauchy when it has a right adjoint module $\Phi^{*}$. Convergent modules are cauchy. Call $\mathscr{C}$ cauchy complete when each cauchy module into $\mathscr{C}$ is convergent.

The cauchy completion $\mathcal{Q} \mathscr{C}$ of a $\mathscr{W}$-category $\mathscr{C}$ is described as follows. The objects over $U$ are cauchy modules $\Phi: U \longrightarrow \mathscr{C}$. The homs are given by:

$$
\mathcal{Q} \mathscr{C}(\Psi, \Phi)=\Psi^{*} \Phi
$$

The Yoneda embedding $Y_{\mathscr{C}}: \mathscr{C} \longrightarrow \mathcal{P} \mathscr{C}$ factors as the composite of two fully faithful functors:

$$
\mathscr{C} \longrightarrow \mathcal{Q} \mathscr{C}, C \mapsto C_{*} \quad ; \quad \mathcal{Q} \mathscr{C} \longrightarrow \mathcal{P} \mathscr{C}, \Phi \mapsto \Phi
$$

A module $\Phi: \mathscr{K} \longrightarrow \mathscr{C}$ is cauchy if and only if the corresponding functor $\mathscr{K} \longrightarrow \mathcal{P} \mathscr{C}$ factors through $\mathcal{Q} \mathscr{C} \longrightarrow \mathcal{P} \mathscr{C}$. Furthermore, $\mathcal{Q} \mathscr{C}$ is cauchy complete with the property that composition with $\mathscr{C} \longrightarrow \mathcal{Q} \mathscr{C}$ determines an equivalence of categories:

$$
\mathscr{W}-\operatorname{Cat}(\mathcal{Q} \mathscr{C}, \mathscr{X}) \simeq \mathscr{W}-\operatorname{Cat}(\mathscr{C}, \mathscr{X})
$$

for all cauchy complete categories $\mathscr{X}$. It follows that the inclusion $\mathcal{Q} \mathscr{C} \longrightarrow \mathcal{Q} \mathscr{C}$ is an equivalence.

If $U, V$ are objects of $\mathscr{W}$, a module from $V$ to $U$ precisely amounts to an arrow $S: V \longrightarrow U$ in $\mathscr{W}$; the module is cauchy if and only if the arrow is a map.

Suppose $\Phi: \mathscr{K} \longrightarrow \mathscr{A}$ is a module. A $\Phi$-indexed colimit for a functor $F: \mathscr{A} \longrightarrow \mathscr{X}$ is a functor, denoted by $\operatorname{colim}(\Phi, F): \mathscr{K} \longrightarrow \mathscr{X}$, together with a 2-cell

$$
\lambda: \Phi \longrightarrow \mathscr{X}(F, \operatorname{colim}(\Phi, F))
$$

which induces an isomorphism

$$
\mathscr{X}(\operatorname{colim}(\Phi, F), X) \cong(\mathcal{P} \mathscr{A})(\Phi, \mathscr{X}(F, X))
$$

of modules from $e X$ to $\mathscr{K}$ for all objects $X$ of $\mathscr{X}$. If the module $F_{*} \Phi$ converges then it converges to $\operatorname{colim}(\Phi, F)$ and the colimit is called absolute. If $\Phi$ is cauchy then $\operatorname{colim}(\Phi, F)$ is absolute whenever it exists (since under those circumstances colim $(\Phi, F)_{*} \cong F_{*} \Phi$ is equivalent to $\operatorname{colim}(\Phi, F)^{*} \cong \Phi^{*} F^{*}$ which is the defining property of colim $\left.(\Phi, F)\right)$.

It follows that there is an isomorphism:

$$
\operatorname{colim}\left(1_{\mathscr{A}}, F\right) \cong F
$$

for all functors $F: \mathscr{A} \longrightarrow \mathscr{X}$, where $1_{\mathscr{A}}$ denotes the identity module of $\mathscr{A}$. 
The colimits indexed by modules $\Psi: U \longrightarrow \mathscr{A}$ with domain in $\mathscr{W}$ are of special importance. Such modules are objects of $\mathcal{P} \mathscr{A}$; and $\operatorname{colim}(\Psi, F)$ is an object of $\mathscr{X}$ over $U$. The reason for this importance is that, provided $\mathscr{X}$ admits sufficient colimits of this special kind, one can construct the more general ones using the formula:

$$
\operatorname{colim}(\Phi, F) K \cong \operatorname{colim}\left(\Phi K_{*}, F\right),
$$

where $\Phi: \mathscr{K} \longrightarrow \mathscr{A}$ is a module, $F: \mathscr{A} \longrightarrow \mathscr{X}$ is a functor and $K$ is an object of $\mathscr{K}$.

A left (Kan) extension $K: \mathscr{B} \longrightarrow \mathscr{X}$ of a functor $F: \mathscr{A} \longrightarrow \mathscr{X}$ along a functor $J: \mathscr{A} \longrightarrow \mathscr{B}$ can be calculated via the formula:

$$
K \cong \operatorname{colim}\left(\mathscr{B}\left(J, 1_{\mathscr{A}}\right), F\right),
$$

when this colimit exists. It is possible for $K$ to exist and for the colimit not to exist; however, such extensions seem to be of little importance. A left extension of $F$ along $J$ is called pointwise when the $\mathscr{B}\left(J, 1_{\mathscr{A}}\right)$-indexed colimit of $F$ exists.

Colimits in $\mathcal{P} \mathscr{C}$ and $\mathcal{P}^{\dagger} \mathscr{C}$ are obtained as follows.

Suppose $\Phi: \mathscr{K} \longrightarrow \mathscr{A}$ is a module. The $\Phi$-indexed colimit of $F: \mathscr{A} \longrightarrow \mathcal{P} \mathscr{C}$ is the functor $\operatorname{colim}(\Phi, F): \mathscr{K} \longrightarrow \mathcal{P} \mathscr{C}$ corresponding to module $\Psi \Phi: \mathscr{K} \longrightarrow \mathscr{C}$ where $\Psi: \mathscr{A} \longrightarrow \mathscr{C}$ is the module corresponding to $F$. The $\Phi$-indexed colimit of $G: \mathscr{A} \longrightarrow \mathcal{P}^{\dagger} \mathscr{C}$ is the functor $\operatorname{colim}(\Phi, G): \mathscr{K} \longrightarrow \mathcal{P}^{\dagger} \mathscr{C}$ corresponding to the module $(\mathcal{P} \mathscr{A})(\Phi, \Gamma)$ : $\mathscr{C} \longrightarrow \mathscr{K}$ where $\Gamma: \mathscr{C} \longrightarrow \mathscr{A}$ is the module corresponding to $G$.

A functor $F: \mathscr{X} \longrightarrow \mathscr{Y}$ is called cocontinuous when it preserves all indexed colimits which exist in $\mathscr{X}$. Each functor with a right adjoint is cocontinuous. Write $\operatorname{Cocts}(\mathscr{X}, \mathscr{Y})$ for the full subcategory of $\mathscr{W}-\operatorname{Cat}(\mathscr{X}, \mathscr{Y})$ consisting of the cocontinuous functors.

Suppose $\mathscr{X}$ admits all colimits indexed by objects of $\mathcal{P} \mathscr{A}$. Then each cocontinuous functor $\mathcal{P} \mathscr{A} \longrightarrow \mathscr{X}$ has a right adjoint and restriction along the Yoneda embedding provides an equivalence of categories:

$$
\operatorname{Cocts}(\mathcal{P} \mathscr{A}, \mathscr{X}) \simeq \mathscr{W}-\operatorname{Cat}(\mathscr{A}, \mathscr{X})
$$

the inverse equivalence takes $F$ to $\operatorname{colim}(-, F)$.

If $\mathscr{X}$ is cauchy complete, and $\Phi$ is cauchy, then $\operatorname{colim}(\Phi, F)$ exists.

A $\mathscr{W}$-category $\mathscr{X}$ is cauchy complete if and only if it admits all colimits indexed by cauchy modules. Thus, if $\mathscr{W}$ has suitably complete and cocomplete hom-categories, both $\mathcal{P} \mathscr{A}$ and $\mathcal{P}^{\dagger} \mathscr{A}$ are cauchy complete. We can use this to prove that $\mathscr{C} \longrightarrow \mathcal{Q} \mathscr{C}$ induces an equivalence:

$$
\mathcal{P Q} \mathscr{C} \simeq \mathcal{P} \mathscr{C}
$$

as the following equivalences show:

$$
\begin{aligned}
\mathscr{W}-\operatorname{Cat}(\mathscr{A}, \mathcal{P} \mathscr{C}) & \simeq \mathscr{W}-\operatorname{Cat}\left(\mathscr{C}, \mathcal{P}^{\dagger} \mathscr{A}\right)^{\mathrm{op}} \\
& \simeq \mathscr{W}-\operatorname{Cat}\left(\mathcal{Q} \mathscr{C}, \mathcal{P}^{\dagger} \mathscr{A}\right)^{\mathrm{op}} \\
& \simeq \mathscr{W}-\operatorname{Cat}(\mathscr{A}, \mathcal{P} \mathcal{Q} \mathscr{C})
\end{aligned}
$$


It can also be seen directly that $\mathscr{C} \longrightarrow \mathcal{Q} \mathscr{C}$ induces an equivalence:

$$
\mathscr{W}-\operatorname{Mod}(\mathscr{A}, \mathcal{Q} \mathscr{C}) \simeq \mathscr{W}-\operatorname{Mod}(\mathscr{A}, \mathscr{C})
$$

provided the composites $\mathscr{A} \longrightarrow \mathscr{C} \longrightarrow \mathcal{Q} \mathscr{C}$ exist.

\section{Torsors and Stacks}

Henceforth suppose that a universe of small sets is given and suppose that the homcategories $\mathscr{W}(U, V)$ have all small limits and small colimits.

A $\mathscr{W}$-category $\mathscr{A}$ will be called small when there exists a small set of objects of $\mathscr{A}$ such that the full sub- $\mathscr{W}$-category $\mathscr{K}$ of $\mathscr{A}$ determined by these objects has the property that the inclusion $\mathscr{K} \longrightarrow \mathscr{A}$ induces an equivalence on cauchy completions.

Using the results of the last two sections we see that, for all small $\mathscr{A}$, both $\mathcal{P} \mathscr{A}$ and $\mathcal{P}^{\dagger} \mathscr{A}$ exist, and modules $\mathscr{C} \longrightarrow \mathscr{A}, \mathscr{A} \longrightarrow \mathscr{X}$ can be composed.

Each functor $F: \mathscr{A} \longrightarrow \mathscr{X}$ determines a $\mathscr{W}$-category $\mathscr{X}[F]$ whose objects are those of $\mathscr{A}$ and whose homs are given by:

$$
\mathscr{X}[F]\left(A^{\prime}, A\right)=\mathscr{X}\left(F A^{\prime}, F A\right) .
$$

Then $F$ factors as the composite of a functor $N_{F}: \mathscr{A} \longrightarrow \mathscr{X}[F]$ bijective on objects, and a fully faithful functor $J_{F}: \mathscr{X}[F] \longrightarrow \mathscr{X}$. If $F$ is fully faithful then $N_{F}$ is an equivalence; and, in this case, $\mathcal{Q} F: \mathcal{Q} \mathscr{A} \longrightarrow \mathcal{Q} \mathscr{X}$ is also fully faithful. If $\mathscr{A}$ is small then $\mathscr{X}[F]$ is small.

Suppose now that we are given certain arrows in $\mathscr{W}$ called covers satisfying the following conditions:

C1. each cover is a map and identities cover;

C2. for each cover $r: V \longrightarrow U$, the diagram

$$
r r^{*} r r^{*} \underset{\varepsilon r r^{*}}{\stackrel{r r^{*} \varepsilon}{\longrightarrow}} r r^{*} \stackrel{\varepsilon}{\longrightarrow} 1_{U}
$$

is a coequalizer in $\mathscr{W}(U, U)$;

C3. if $r: V \longrightarrow U$ is a cover and $f: W \longrightarrow U$ is a map, then there exist a cover $r^{\prime}: V^{\prime} \longrightarrow W$ and a map $f^{\prime}: V^{\prime} \longrightarrow V$ such that $r f^{\prime} \cong f r^{\prime}$;

C4. if $s r \cong t$ for maps $r, s, t$ with $r$ a cover then $s$ is a cover precisely when $t$ is.

It follows from $\mathrm{C} 2$. that, for all covers $r: V \longrightarrow U$ and all $\mathscr{W}$-categories $\mathscr{X}$, the functor

$$
\mathscr{W}-\operatorname{Mod}(r, \mathscr{X}): \mathscr{W}-\operatorname{Mod}(U, \mathscr{X}) \longrightarrow \mathscr{W}-\operatorname{Mod}(V, \mathscr{X})
$$


reflects isomorphisms.

Suppose $F: \mathscr{A} \longrightarrow \mathscr{X}$ is a functor. An object $X$ of $\mathscr{X}$ over $U$ is said to be locally isomorphic to a value of $F$ when there exist a cover $r: V \longrightarrow U$ and an object $A$ of $\mathscr{A}$ over $V$ such that $F A \cong \operatorname{colim}(r, X)$.

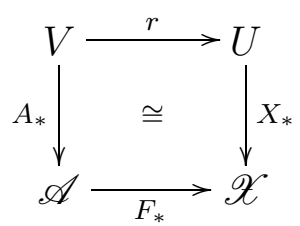

Write $\operatorname{Loc}_{\mathscr{X}}(F)$ for the full sub- $\mathscr{W}$-category of $\mathscr{X}$ consisting of those objects which are locally isomorphic to a value of $F$. We say that $F$ is a local equivalence when it is fully faithful and $\operatorname{Loc}_{\mathscr{X}}(F)=\mathscr{X}$. Notice that

$$
\operatorname{Loc}_{\mathscr{X}}(F)=\operatorname{Loc}_{\mathscr{X}}\left(J_{F}\right)
$$

so, if $\operatorname{Loc}_{\mathscr{X}}(F)=\mathscr{X}$ then $J_{F}$ is a local equivalence.

For a small $\mathscr{W}$-category $\mathscr{A}$, we define an $\mathscr{A}$-torsor to be an object of $\mathcal{P} \mathscr{A}$ which is locally isomorphic to a value of the Yoneda embedding $Y_{\mathscr{A}}: \mathscr{A} \longrightarrow \mathcal{P} \mathscr{A}$. In other words, a module $\Phi: U \longrightarrow \mathscr{A}$ is a torsor when it is locally convergent.

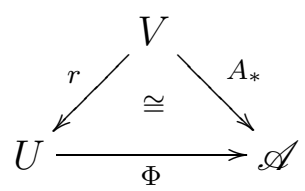

Put Tor $\mathscr{A}=\operatorname{Loc}_{\mathcal{P} \mathscr{A}}\left(Y_{\mathscr{A}}\right)$.

5.1. Proposition. Each torsor is a cauchy module. So Tor $\mathscr{A} \subset \mathcal{Q} \mathscr{A}$.

Proof. For each $\mathscr{W}$-category $\mathscr{X}$, consider the triangle:

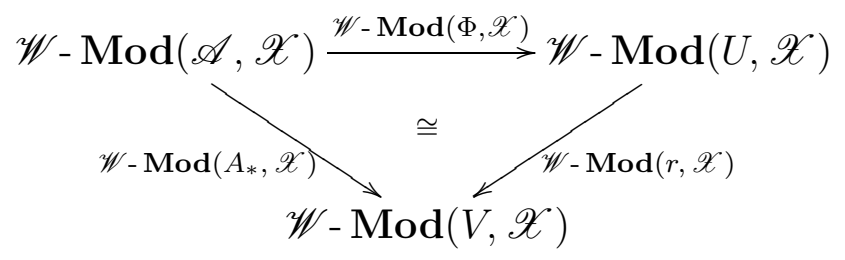

The downward arrows have left adjoints and the counit of the one on the right-hand side is a regular epimorphism. Since $\mathscr{W}-\operatorname{Mod}(\mathscr{A}, \mathscr{X})$ has coequalizers; it follows that $\mathscr{W}-\operatorname{Mod}(\Phi, \mathscr{X})$ has a left adjoint. Hence $\Phi$ is cauchy.

A $\mathscr{W}$-category $\mathscr{X}$ is called a stack when it admits all colimits indexed by torsors. 


\subsection{Proposition.}

(a) If $X$ is locally isomorphic to a value of a fully faithful functor $F: \mathscr{A} \longrightarrow \mathscr{X}$ then $\mathscr{X}(F, X)$ is an $\mathscr{A}$-torsor.

(b) If $\Phi$ is an $\mathscr{A}$-torsor and $F: \mathscr{A} \longrightarrow \mathscr{X}$ is any functor into a stack $\mathscr{X}$ then $\operatorname{colim}(\Phi, F)$ is locally isomorphic to a value of $F$.

(c) If $\mathscr{X}$ is a stack then restriction along any local equivalence $G: \mathscr{A} \longrightarrow \mathscr{C}$ yields an equivalence:

$$
\mathscr{W}-\operatorname{Cat}(\mathscr{C}, \mathscr{X}) \simeq \mathscr{W}-\operatorname{Cat}(\mathscr{A}, \mathscr{X})
$$

PROOF.

(a) If $F A \cong \operatorname{colim}(r, X)$ then:

$$
\mathscr{X}(F, X) r \cong \mathscr{X}(F, 1) X_{*} r \cong \mathscr{X}(F, 1)(F A)_{*} \cong \mathscr{X}(F, F) A_{*} \cong A_{*}
$$

since $F$ is fully faithful.

(b) If $\Phi r \cong A_{*}$ then;

$$
\operatorname{colim}(r, \operatorname{colim}(\Phi, F))_{*} \cong \operatorname{colim}(\Phi, F)_{*} r \cong F_{*} \Phi r \cong F_{*} A_{*} \cong(F A)_{*} .
$$

(c) For each $F: \mathscr{A} \longrightarrow \mathscr{X}$, the pointwise left extension $K$ of $F$ along $G$ is given by $K \cong \operatorname{colim}(\mathscr{C}(G, 1), F)$; it exists by (a) since every object of $\mathscr{C}$ is locally isomorphic to a value of $G$. Since $G$ is fully faithful, $F \cong K G$. Thus $F \mapsto K$ gives the inverse equivalence.

5.3. TheOrem. [Classification property of torsors] If $F: \mathscr{A} \longrightarrow \mathscr{X}$ is a $\mathscr{W}$-functor with $\mathscr{A}$ small and $\mathscr{X}$ a stack then the $\mathscr{W}$-functor $\mathscr{X} \longrightarrow \mathcal{P} \mathscr{X}[F]$ corresponding to $J_{F}$ induces an equivalence:

$$
\operatorname{Loc}_{\mathscr{X}}(F) \simeq \operatorname{Tor} \mathscr{X}[F] .
$$

Proof. A left adjoint to $\mathscr{X} \longrightarrow \mathcal{P} \mathscr{X}[F]$ at $\Phi$ is a $\operatorname{colim}\left(\Phi, J_{F}\right)$ which exists when $\Phi$ is a torsor since $\mathscr{X}$ is a stack. By Proposition 5.2 this adjunction restricts to an adjunction between $\operatorname{Loc}_{\mathscr{X}}(F)$ and Tor $\mathscr{X}[F]$. Since $J_{F}$ is fully faithful, the unit of this adjunction is an isomorphism. It remains to show that the counit $\operatorname{colim}\left(\mathscr{X}\left(J_{F}, X\right), J_{F}\right) \longrightarrow X$ is an isomorphism when $\operatorname{colim}(r, X) \cong F A$ for some cover $r$. Since composition on the right with $r$ reflects isomorphisms, the following calculation completes the proof:

$$
\begin{aligned}
\operatorname{colim}\left(\mathscr{X}\left(J_{F}, X\right), J_{F}\right)_{*} r & \cong J_{F^{*}} J_{F}^{*} X_{*} r \\
& \cong J_{F^{*}} J_{F}^{*} F_{*} A_{*} \\
& \cong J_{F^{*}} J_{F}^{*} J_{F^{*}} N_{F^{*}} A_{*} \\
& \cong F_{*} A_{*} \\
& \cong X_{*} r .
\end{aligned}
$$


5.4. Theorem. For each small $\mathscr{W}$-category $\mathscr{C}$, the $\mathscr{W}$-category Tor $\mathscr{C}$ is the associated stack. That is, Tor $\mathscr{C}$ is a stack and the embedding $\mathscr{C} \longrightarrow$ Tor $\mathscr{C}$ induces an equivalence

$$
\mathscr{W}-\operatorname{Cat}(\operatorname{Tor} \mathscr{C}, \mathscr{X}) \simeq \mathscr{W}-\operatorname{Cat}(\mathscr{C}, \mathscr{X})
$$

for all stacks $\mathscr{X}$.

Proof. Clearly $\mathscr{C} \longrightarrow$ Tor $\mathscr{C}$ is a local equivalence so, from Proposition 5.2(c), all that remains to be shown is that $\operatorname{Tor} \mathscr{C}$ is a stack. Suppose $\Phi: U \longrightarrow \mathscr{C}$ is a torsor with $\Phi r=$ $A_{*}$, and suppose $G: \mathscr{A} \longrightarrow \operatorname{Tor} \mathscr{C}$ is a functor. We must show that $\operatorname{colim}(\Phi, G)$ exists. Let $\Gamma: \mathscr{A} \longrightarrow \mathscr{C}$ denote the module corresponding to the functor $\mathscr{A} \stackrel{G}{\longrightarrow}$ Tor $\mathscr{C} \longrightarrow \mathcal{P} \mathscr{C}$. The $\Phi$-indexed colimit of the last functor is $\Gamma \Phi$. We must show that $\Gamma \Phi$ is in fact a $\mathscr{C}-$ torsor. But $\Gamma A_{*}$ is the $\mathscr{C}$-torsor $G A$. So there is a cover $s: W \longrightarrow V$ with $\Gamma A_{*} s \cong C_{*}$, and we have $(\Gamma \Phi)(r s) \cong \Gamma A_{*} s \cong C_{*}$. Using C4., we have that $\Gamma \Phi$ is a torsor.

A $\mathscr{W}$-category $\mathscr{A}$ is called map-tensored when, for all maps $f: V \longrightarrow U$ in $\mathscr{W}$ and all objects $A$ of $\mathscr{A}$ over $U$, the module $A_{*} f$ is convergent; that is, $\operatorname{colim}(f, A)$ exists. Since $f$ has a right adjoint, if $\mathscr{A}$ is cauchy complete then $\mathscr{A}$ is map-tensored. If $\mathscr{A}$ is map-tensored then so is Tor $\mathscr{A}$ (using C3.).

The map-tensor cocompletion $\mathcal{M} \mathscr{A}$ of $\mathscr{A}$ is described as follows. The objects over $U$ are pairs $(a, A)$ where $a: U \longrightarrow V$ is a map in $\mathscr{W}$ and $A$ is an object of $\mathscr{A}$ over $V$. The homs are given by:

$$
(\mathcal{M} \mathscr{A})((b, B),(a, A))=b^{*} \mathscr{A}(B, A) a .
$$

There are fully faithful functors:

$$
M_{\mathscr{A}}: \mathscr{A} \longrightarrow \mathcal{M} \mathscr{A}, A \mapsto(1, A) \quad ; \quad \mathcal{M} \mathscr{A} \longrightarrow \mathcal{Q} \mathscr{A},(a, A) \mapsto A_{*} a .
$$

Note that $\mathscr{A}$ is map-tensored if and only if $M_{\mathscr{A}}$ has a left adjoint; and that $\mathcal{M} \mathscr{A}$ is map-tensored. Clearly $M_{\mathscr{A}}$ induces an equivalence $\mathcal{Q} \mathscr{A} \simeq \mathcal{Q M} \mathscr{A}$, so Tor $\mathcal{M} \mathscr{A}$ can be identified with a full sub- $\mathscr{W}$-category of $\mathcal{Q} \mathscr{A}$. To be specific, an object $\Phi: U \longrightarrow \mathscr{A}$ of $\mathcal{Q} \mathscr{A}$ is an $\mathcal{M} \mathscr{A}$-torsor if and only if there exist a cover $r: V \longrightarrow U$, a map $a: V \longrightarrow W$, and an object $A$ of $\mathscr{A}$ over $W$ such that:

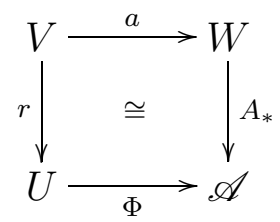

A module $\Phi: \mathscr{C} \longrightarrow \mathscr{A}$ is called weakly convergent when there exists a local equivalence $G: \mathscr{K} \longrightarrow \mathcal{M} \mathscr{C}$ such that $\Phi M_{\mathscr{C}}^{*} G_{*}: \mathscr{K} \longrightarrow \mathscr{A}$ is convergent. We write $\mathbf{W c g t}(\mathscr{C}, \mathscr{A})$ for the full subcategory of $\mathscr{W}-\operatorname{Mod}(\mathscr{C}, \mathscr{A})$ consisting of the weakly convergent modules.

If $\mathscr{A}$ is map-tensored then every convergent module into $\mathscr{A}$ is weakly convergent.

Since $M_{\mathscr{C}}$ is fully faithful, one easily sees that, if $\Phi$ is weakly convergent, the corresponding functor $\mathscr{C} \longrightarrow \mathcal{P} \mathscr{A}$ factors through Tor $\mathscr{A} \longrightarrow \mathcal{P} \mathscr{A}$. So we always have a fully faithful functor

$$
\operatorname{Wcgt}(\mathscr{C}, \mathscr{A}) \longrightarrow \mathscr{W}-\operatorname{Cat}(\mathscr{C}, \text { Tor } \mathscr{A})
$$


5.5. Proposition. [Cocycle property of torsors] If $\mathscr{A}$ is map-tensored then the last functor is an equivalence

$$
\operatorname{Wcgt}(\mathscr{C}, \mathscr{A}) \simeq \mathscr{W}-\operatorname{Cat}(\mathscr{C}, \text { Tor } \mathscr{A})
$$

Proof. Given a functor $F: \mathscr{C} \longrightarrow$ Tor $\mathscr{A}$, for each $C$ in $\mathscr{C}$, we have a cover $r_{C}$ : $V_{C} \longrightarrow e C$ and $A_{C}$ in $\mathscr{A}$ over $V_{C}$ with $(F C) r_{C} \cong A_{C^{*}}$. Consider the full sub- $\mathscr{W}$-category $\mathscr{R}$ of $\mathcal{M} \mathscr{C}$ consisting of the objects $\left(r_{C}, C\right)$. The inclusion induces a fully faithful maptensor-preserving functor $\mathcal{M} \mathscr{R} \longrightarrow \mathcal{M} \mathscr{C}$ which, using $\mathrm{C} 3$., is a local equivalence. The effect of $F$ on homs gives 2-cells:

$$
C^{*} C_{*} \longrightarrow\left(F C^{\prime}\right)^{*}(F C)_{*} .
$$

Applying $r_{C^{\prime}}^{*}$ on the left and $r_{C}$ on the right, we obtain 2-cells

$$
r_{C^{\prime}}^{*} \mathscr{C}\left(C^{\prime}, C\right) r_{C} \longrightarrow \mathscr{A}\left(A_{C^{\prime}}, A_{C}\right) .
$$

This gives the effect on homs of a functor $\mathscr{R} \longrightarrow \mathscr{A}$ taking $\left(r_{C}, C\right)$ to $A_{C}$. Since $\mathscr{A}$ is map-tensored there is a map-tensor-preserving extension $\mathcal{M} \mathscr{R} \longrightarrow \mathscr{A}$ to which the composite

$$
\mathcal{M} \mathscr{R} \longrightarrow \mathcal{M} \mathscr{C} \stackrel{M_{C}^{*}}{\longrightarrow} \mathscr{C} \stackrel{\Phi}{\longrightarrow} \mathscr{A}
$$

converges, where $\Phi$ is the module corresponding to $F$.

5.6. Example. Return to Example 2.1 where $\mathscr{W}=\mathbf{S p n} \mathscr{E}$ with $\mathscr{E}$ small complete and small cocomplete. We have extended the theory of torsors and stacks based on $\mathscr{E}$ as appearing in Giraud [Gir], Bunge [Bun], Bourn [Bou], Street [St3].

Acknowledgments. I would like to thank Renato Betti for helpful conversations and for the hospitality of the Università di Milano during the preparation of this manuscript. Thanks are also due to André Joyal for discussions in Australia on torsors. The basic example of an enriched category arising from a locally small fibration was observed by R.F.C. Walters after a seminar in which the author defined locally small fibrations using the two-sided characterization of [St2, Proposition (9.3)(ii)].

\section{References}

[Bé1] J. Bénabou, Introduction to bicategories, Lecture Notes in Math., 47 (Springer, Berlin,1967), 1-77.

[Bé2] J. Bénabou, Fibrations petites et localement petites, C.R. Acad. Sci. Paris Sér.A, 281 (1975), 897-900.

[Bt1] R. Betti, Una teoria categoriale degli automi, Università degli Studi di Milano, Instituto Mathematico "F. Enriques", Quaderno n. 35/s (1979). 
[Bt2] R. Betti, Bicategorie di base, Università degli Studi di Milano, Istituto Mathematico "F. Enriques", Quaderno n. 2/s (II) (1981).

[BC] R. Betti and A. Carboni, Cauchy completion and associated sheaf, Università degli Studi di Milano, Istituto Mathematico "F. Enriques", Quaderno n. 5/p (II) (1981).

[Bou] D. Bourn, Une autre propriété universelle pour le champ associé, Cahiers de Topologie et Géométrie Différentielle, XXI - 4 (1980), 403-410.

[Bun] M. Bunge, Stack completions and Morita equivalence for categories in a topos, Cahiers de Topologie et Géométrie Différentielle, XX - 4 (1979), 401-436.

[Day] B. Day, Limit spaces and closed span categories, Lecture Notes in Math., 420 (Springer, Berlin - N.Y.,1974), 65-74.

[EK] S. Eilenberg and G.M. Kelly, Closed categories, Proc. Conf. Categorical Algebra at La Jolla, (Springer, Berlin - N.Y.,1966), 421-562.

[Gir] J. Giraud, Cohomologie non abélienne, (Springer, Berlin,1971).

[Law] F.W. Lawvere, Teoria delle categorie sopra un topos di base, Lecture Notes, Univ. of Perugia, (1973).

[PS] R. Paré and D. Schumacher, Abstract families and the adjoint functor theorem, Lecture Notes in Math., 661 (Springer, Berlin, 1978), 1-125.

[Pen] J. Penon, Catégories localement internes, C. R. Acad. Sci. Paris Sér. A, 278 (1974), 1577-1580.

[St1] R.H. Street, Elementary cosmoi, Lecture Notes in Math., 420 (Springer, Berlin,1974), 134-180.

[St2] R.H. Street, Cosmoi of internal categories, Transactions Amer. Math. Soc., 258 (1980), 271-318.

[St3] R.H. Street, Two dimensional sheaf theory, J. Pure and Applied Algebra, 23(3), (1982), 251-270.

[SW] R.H. Street and R.F.C. Walters, Yoneda structures on 2-categories, J. Algebra, 50 (1978), 350-379.

[Wal] R.F.C. Walters, Sheaves on sites as Cauchy-complete categories, J. Pure and Applied Algebra, 24(1) (1982), 95-102. 
Department of Mathematics, Macquarie University NSW 2109, Australia

Email: street@math.mq.edu.au

This article may be accessed from http://www.tac.mta.ca/tac/reprints or by anonymous ftp at

ftp://ftp.tac.mta.ca/pub/tac/html/tac/reprints/articles/14/tr14.\{dvi,ps\} 
REPRINTS IN THEORY AND APPLICATIONS OF CATEGORIES will disseminate articles from the body of important literature in Category Theory and closely related subjects which have never been published in journal form, or which have been published in journals whose narrow circulation makes access very difficult. Publication in 'Reprints in Theory and Applications of Categories' will permit free and full dissemination of such documents over the Internet. Articles appearing have been critically reviewed by the Editorial Board of Theory and Applications of Categories. Only articles of lasting significance are considered for publication. Distribution is via the Internet tools WWW/ftp.

SUBSCRIPTION INFORMATION. Individual subscribers receive (by e-mail) abstracts of articles as they are published. To subscribe, send e-mail to tac@mta.ca including a full name and postal address. For institutional subscription, send enquiries to the Managing Editor.

SELECTION OF REPRINTS. After obtaining written permission from any copyright holder, any three TAC Editors may propose a work for TAC Reprints to the Managing Editor. The proposal will be reported to all Editors. The Managing Editor may either accept the proposal or require that the Editors vote on it. Before such a vote, the author, title and original publication data will be circulated to Editors. If a $2 / 3$ majority of those TAC Editors responding within one month agrees, the work will be accepted for TAC Reprints. After a work is accepted, the author or proposer must provide to TAC either a usable TeX source or a PDF document acceptable to the Managing Editor that reproduces a typeset version. Up to five pages of corrections, commentary and forward pointers may be appended by the author. When submitting commentary, authors should read and follow the Format for submission of Theory and Applications of Categories at http://www.tac.mta.ca/tac/.

Managing editor. Robert Rosebrugh, Mount Allison University: rrosebrugh@mta.ca

TEXnical editor. Michael Barr, McGill University: mbarr@barrs .org

Transmitting editors.

Richard Blute, Université d' Ottawa: rblute@mathstat.uottawa.ca

Lawrence Breen, Université de Paris 13: breen@math.univ-paris13.fr

Ronald Brown, University of North Wales: r.brown@bangor.ac.uk

Jean-Luc Brylinski, Pennsylvania State University: jlb@math.psu.edu

Aurelio Carboni, Università dell Insubria: aurelio.carboni@uninsubria.it

Valeria de Paiva, Xerox Palo Alto Research Center: paiva@parc.xerox.com

Ezra Getzler, Northwestern University: getzler (at)math (dot)northwestern (dot)edu

Martin Hyland, University of Cambridge: M.Hyland@dpmms.cam.ac.uk

P. T. Johnstone, University of Cambridge: ptj@dpmms.cam.ac.uk

G. Max Kelly, University of Sydney: maxk@maths.usyd.edu.au

Anders Kock, University of Aarhus: kock@imf .au.dk

Stephen Lack, University of Western Sydney: s.lack@uws.edu.au

F. William Lawvere, State University of New York at Buffalo: wlawvere@acsu .buffalo.edu

Jean-Louis Loday, Université de Strasbourg: loday@math.u-strasbg.fr

Ieke Moerdijk, University of Utrecht: moerdijk@math.uu.nl

Susan Niefield, Union College: niefiels@union.edu

Robert Paré, Dalhousie University: pare@mathstat.dal.ca

Jiri Rosicky, Masaryk University: rosicky@math.muni.cz

Brooke Shipley, University of Illinois at Chicago: bshipley@math.uic.edu

James Stasheff, University of North Carolina: jds@math.unc.edu

Ross Street, Macquarie University: street@math.mq.edu .au

Walter Tholen, York University: tholen@mathstat.yorku.ca

Myles Tierney, Rutgers University: tierney@math.rutgers.edu

Robert F. C. Walters, University of Insubria: robert.walters@uninsubria.it

R. J. Wood, Dalhousie University: rjwood@mathstat.dal.ca 